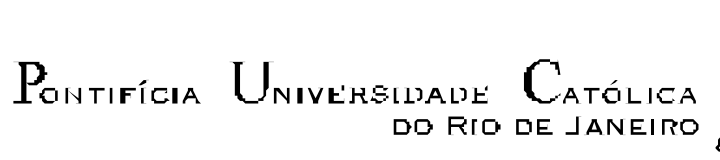

Gabriel Buchmann

Interação entre Educação, Fecundidade e Economia Política e suas conseqüências para a Distribuição de Renda

Dissertação apresentada como requisito parcial para obtenção do título de Mestre pelo Programa de PósGraduação em Economia da PUC-Rio.

Orientador: Rodrigo Reis Soares

Rio de Janeiro Agosto de 2007 


$$
\text { Pontifícia U Jiveraidade Católiga }
$$

Gabriel Buchmann

\title{
Interação entre Educação, Fecundidade e Economia \\ Política e suas conseqüências para a \\ Distribuição de Renda
}

\begin{abstract}
Dissertação apresentada como requisito parcial para obtenção do título de Mestre pelo Programa de Pós-Graduação em Economia da PUC-Rio. Aprovada pela Comissão Examinadora abaixo assinada.
\end{abstract}

\author{
Rodrigo Reis Soares \\ Orientador \\ PUC-Rio \\ Juliano Junqueira Assunção \\ PUC-Rio \\ Marcelo Côrtes Neri \\ EPGE / IBRE - FGV-Rio \\ João Pontes Nogueira
}

Coordenador Setorial do Centro de Ciências Sociais - PUC-Rio

Rio de Janeiro, 27 de agosto de 2007 
Todos os direitos reservados. É proibida a reprodução total ou parcial do trabalho sem autorização da universidade, do autor e do orientador.

\section{Gabriel Buchmann}

Graduou-se em Ciências Econômicas pela PUC-Rio, tendo cursado um semestre na Universidad Autónoma de Madrid e um semestre no Institute de Sciences Politiques de Paris. Especializou-se também em Relações Internacionais pelo IRI/PUC-Rio.

Ficha Catalográfica

Buchmann, Gabriel

Interação entre Educação, Fecundidade e Economia Política e suas conseqüências para a Distribuição de Renda / Gabriel Buchmann; orientador: Rodrigo Reis Soares. - Rio de Janeiro: PUC-Rio, Departamento de Economia, 2007.

v., $61 \mathrm{f:}$ il. ; $29,7 \mathrm{~cm}$

1. Dissertação (mestrado) - Pontifícia Universidade Católica do Rio de Janeiro, Departamento de Economia.

Inclui referências bibliográficas.

1. Economia - Tese. 2. Educação. 3. Fecundidade. 4. Economia Política . 5. Distribuição de Renda. 6. Modelagem Teórica com Simulações Numéricas. I. Soares, Rodrigo Reis. II. Pontifícia Universidade Católica do Rio de Janeiro. Departamento de Economia. III. Título. 
Essa dissertação é dedicada a mamãe e papai, meus grandes heróis, a minha querida Vó Zinda, ao meu primo Ricardinho, a toda a minha família e amigos, que são a família que a gente escolhe, e a Ilana, por todo o apoio e incentivo. 


\section{Agradecimentos}

Rodrigo, pela brilhante orientação, sem dúvida a melhor que eu poderia ter tido e sem a qual nada disso seria possível,

Felipe Cesar Diósgenes, Antonio Sodré e Pedro James Hemsley, pela coorientação pró-forma,

Juan Pablo e Marcos Castro, pela ajuda em tentar resolver o modelo analiticamente,

os membros da minha banca Juliano e Marcelo pelas valiosas contribuições,

e todos os professores que ao longo da minha vida me proporcionaram o capital humano necessário para confecção deste trabalho.

Também Fernando Bignotto, pelas fundamentais e enumeráveis ajudas com o latex, minha ferramenta de trabalho, e o próprio latex.

Letícia Frossard, Juliana Salomão, Mamãe e Nina, pela revisão e tradução de ambas as versões desta dissertação.

Todos os colegas com os quais convivi intensamente durante este mestrado, desta que foi a melhor turma que tive ao longo da vida,

e todo o departamento de economia.

E, por último, a Mamãe, Vó Zinda, Joaquim e sogros Chico de Assis, Sandra e Júlio por todo o apoio e estrutura que me proporcionaram durante a confecção deste trabalho, também sem o quais nada disso seria possível.

E a querida favelinha, que me acolheu como segunda casa durante esses últimos dois anos. 


\section{Resumo}

Buchmann, Gabriel; Soares, Rodrigo Reis. Interação entre Educação, Fecundidade e Economia Política e suas conseqüências para a Distribuição de Renda. Rio de Janeiro, 2007. 61p. Dissertação de Mestrado — Departamento de Economia, Pontifícia Universidade Católica do Rio de Janeiro.

Este trabalho constrói um modelo que gera uma dinâmica onde interagem (i) decisões educacionais, tanto individuais, quanto no nível público, (ii) decisões da fecundidade, e (iii) a economia política de uma sociedade. Esta interação, em conseqüência, determina: (i) a qualidade relativa do ensino público e privado e sua distribuição, (ii) o diferencial de fecundidade entre grupos sociais e (iii) a distribuição do poder político que, conjuntamente, determinam a evolução da distribuição de renda na sociedade. Apesar de ser um modelo geral, busca-se a adequação a alguns fatos estilizados e a evidências empíricas encontradas no Brasil, um país muito desigual, com um sistema democrático relativamente novo e com sérios problemas educacionais Resolvo então o equilíbrio estático e calibro os parâmetros, resolvendo a dinâmica numericamente. Mostro que, se a democracia funcionar bem, teremos um equilíbrio sem desigualdade no longo prazo, e explico então as forças que nos mantêm em uma armadilha de desigualdade elevada.

\section{Palavras-chave}

Educação. Fecundidade. Economia Política . Distribuição de Renda. Modelagem Teórica com Simulações Numéricas. 


\section{Abstract}

Buchmann, Gabriel; Soares, Rodrigo Reis. . Rio de Janeiro, 2007. 61p. MsC Thesis - Department of Economia, Pontifícia Universidade Católica do Rio de Janeiro.

This paper builds a model whose main idea is to generate a dynamics in which (i) educational decisions, at the individual as well at the public level, (ii) fertility decisions, and (iii) the political economy of a society interact and determine the (i) relative quality of public and private education and its distribution, (ii)the fertility differential between the groups and (iii) the distribution of political power, which jointly shape the evolution of income distribution in society. In spite of being a general model, it fits some stylized facts and empirical evidence found in Brazil, a very unequal country with a quite young democracy and very serious educational problems. We solve for the static equilibrium and then calibrate the parameters and solve it numerically. We find that if democracy works well, then we will have no inequality in the long run, and explain which are the forces that maintain us in a high inequality trap.

\section{Keywords}

Education. Fertility. Political Economy. Income Distribution. Theoretical Model with Numeric Simulations. 


\section{Sumário}

$\begin{array}{lll}1 & \text { Introdução } & \mathbf{1 0}\end{array}$

\begin{tabular}{lll}
\hline 1.1 & Motivação - O Caso Brasileiro & 13
\end{tabular}

\begin{tabular}{lll}
\hline 1.2 & Literatura Relacionada & 15
\end{tabular}

\begin{tabular}{lll}
\hline 2 & O Modelo & 19
\end{tabular}

$\begin{array}{lll}3 & \text { Resolvendo o modelo } & 24\end{array}$

3.1 Escolha da Fecundidade 24

3.2 Escolha Educacional 26

$\begin{array}{lll}3.3 & \text { Escolha do Imposto } & 27\end{array}$

$4 \quad$ Modelo Político e Equilíbrio Estático 31

\begin{tabular}{lll}
\hline 4.1 & Democracia & 31
\end{tabular}

$\begin{array}{lll}4.2 & \text { Plutocracia } & 32\end{array}$

5 Simulando a Dinâmica 34

$\begin{array}{lll}5.1 & \text { Calibragem } & 36\end{array}$

5.2 Resultado das Simulações - Democracia 37

5.3 Resultado das Simulações - Plutocracia 43

5.4 Um Caso Idílico 48

5.5 Estática Comparativa 49

\begin{tabular}{lll}
\hline Conclusão & 51
\end{tabular}

\begin{tabular}{ll}
\hline Referências Bibliográficas & 53
\end{tabular}

\begin{tabular}{lll}
\hline A & Bases de Dados & 57
\end{tabular}

\begin{tabular}{lll}
\hline B Um Caso Mais Geral & 58
\end{tabular}

\begin{tabular}{lll}
\hline C & Outros Gráficos & 61
\end{tabular} 


\section{Lista de figuras}

5.1 Evolução dos Equilíbrios 37

5.2 Evolução do Imposto e da Qualidade da Educação Pública 38

5.3 Diferencial de Fecundidade e Crescimento Demográfico 39

5.4 Medidas de Desigualdade 40

5.5 Renda Agregada e Renda per capita 40

5.6 Evolução dos Equilíbrios, do Imposto e da Qualidade da Educação Pública

5.7 Diferencial de Fecundidade e Medidas de Desigualdade 42

$5.8 \quad P_{t}^{p u b}=55 \%$ e $P_{t}^{\text {priv }}=45 \%$ vs $P_{t}^{\text {pub }}=85 \%$ e $P_{t}^{\text {priv }}=15 \%$

5.9 Evolução dos Equilíbrios 43

5.10 Evolução do Imposto e da Qualidade da Educação Pública 44

5.11 Diferencial de Fecundidade e Crescimento Demográfico 44

5.12 Medidas de Desigualdade 45

5.13 Renda Agregada e Renda per capita 46

5.14 Evolução dos Equilíbrios e Evolução do Imposto 46

5.15 Qualidade da Educação Pública e Medidas de Desigualdade 47

$5.16 P_{t}^{p u b}=55 \%$ e $P_{t}^{p r i v}=45 \%$ vs $P_{t}^{p u b}=85 \%$ e $P_{t}^{\text {priv }}=15 \% \quad 47$

$5.17 \phi=\mathbf{0 , 2}$ e $\phi=\mathbf{0 , 3} \quad 49$

$5.18 \pi=5$ e $\pi=6$

$5.19 \phi=\mathbf{0 , 2}$ e $\phi=0,3$ e $\quad 50$

$5.20 \pi=4$ e $\pi=5$

C.1 Alocação da Renda Pessoal - Democracia - CH pub vs CH priv 61

C.2 Alocação da Renda Pessoal - Plutocracia - CH pub vs CH priv 